\title{
Uncertainty Modeling and Evaluation of CMM Task Oriented Measurement Based on SVCMM
}

\author{
Hongli Li ${ }^{1,2}$, Xiaohuai Chen ${ }^{1}$, Yinbao Cheng ${ }^{1}$, Houde Liu ${ }^{2}$, Hanbin Wang ${ }^{3}$, Zhenying Cheng ${ }^{1}$, \\ Hongtao Wang ${ }^{1}$ \\ ${ }^{1}$ School of Instrument Science and Opto-electronics Engineering, Hefei University of Technology, Tunxi Rd 193, Hefei \\ 230009,China,lihongli_hgd@163.com/hlli@hfut.edu.cn \\ 2 Shenzhen Engineering Laboratory of Geometry Measurement Technology, Graduate School at Shenzhen, Tsinghua \\ University, Shenzhen 518055, China \\ ${ }^{3}$ Fujian Metrology Institute, Fuzhou 350003, China
}

\begin{abstract}
Due to the variety of measurement tasks and the complexity of the errors of coordinate measuring machine (CMM), it is very difficult to reasonably evaluate the uncertainty of the measurement results of CMM. It has limited the application of CMM. Task oriented uncertainty evaluation has become a difficult problem to be solved. Taking dimension measurement as an example, this paper puts forward a practical method of uncertainty modeling and evaluation of CMM task oriented measurement (called SVCMM method). This method makes full use of the CMM acceptance or reinspection report and the Monte Carlo computer simulation method (MCM). The evaluation example is presented, and the results are evaluated by the traditional method given in GUM and the proposed method, respectively. The SVCMM method is verified to be feasible and practical. It can help CMM users to conveniently complete the measurement uncertainty evaluation through a single measurement cycle.
\end{abstract}

Keywords: CMM, uncertainty modeling, uncertainty evaluation, MCM, SVCMM.

\section{INTRODUCTION}

The new generation Geometrical Product Specifications (GPS) requests for providing the standard uncertainty reports in product inspection and laboratory certification [1], [2]. While in the current practical applications, as indispensable and important equipment in manufacturing and product inspection, coordinate measuring machine (CMM) usually can only give the estimate of the parameters to be measured, but cannot provide the uncertainty of the measurement results [3], [4]. For the CMM users, it is difficult to complete uncertainty modeling and evaluation of CMM task oriented measurement.

The evaluation method of the sensitivity coefficient analysis and square-sum-root synthesis has been given out in the Guide to the Expression of Uncertainty in Measurement (GUM) [5], which is called GUM method in this paper for convenient expression. Although it has been widely recognized in the industry, it is usually difficult to implement in practice.

It is difficult to establish the measurement models because the CMM measurement tasks are complex and diverse. Especially for the complex or non-linear measurement model, it is very difficult to calculate the transfer coefficient and investigate the correlation between the inputs based on the GUM method, the evaluation staff needs professional knowledge about uncertainty evaluation, and extensive assessment experience [6], [7]. However, it is a practical method to evaluate uncertainty based on statistics by computer simulation [8], [9]. Evaluating measurement uncertainty by computer simulation has application prospect [10], [11], [12], [13], [14].

Taking dimension measurement as an example, this paper presents a practical method for uncertainty modeling and evaluation of CMM task oriented measurement, and also gives an evaluation example.

In this method, the measurement uncertainty analysis model is established based on measurement system analysis, and it is easy to understand and expand. Moreover, the method makes full use of the CMM acceptance or reinspection report as well as expert experience as constraint condition, and flexibly uses the Monte Carlo computer simulation (MCM) to evaluate measurement uncertainty. It is a simplified virtual coordinate measuring machine, which is abbreviated to SVCMM in this paper. The validity and practicability of this method can be demonstrated by the evaluation example.

The CMM users only need to complete a single measurement process and can realize the uncertainty evaluation of CMM task oriented measurement conveniently and effectively based on the SVCMM method. 


\section{MEASUREMENT UNCERTAINTY MODELING}

For the dimensional measurement tasks of CMM, a general measurement model can be expressed as a function related to the coordinates of sampling points.

$$
Y=g\left(x, y, z, a_{1}, a_{2}, \ldots, a_{n}\right)
$$

Where, $Y$ is the result calculated by the measurement model, $x, y, z$ are the measurement point coordinates, $a_{i}$ is the variable parameter.

In the actual measurement process, there are many factors that will affect the measurement results. The main sources of measurement uncertainty include the CMM indication error, measurement repeatability, measurement reproducibility, workpieces inconsistence, temperature variation, and measurement speed. The main uncertainty sources are relatively independent. Among them, the temperature variation will not only bring the uncertainty of temperature compensation, but also change the thermal expansion coefficient of the measured workpiece and the thermal expansion coefficient of CMM grating ruler. For the contact measurement, the influence of workpieces inconsistence is mainly manifested as that the workpiece shape deviation or geometric deviation will affect the measurement results. The measurement speed will affect the measuring force. If the CMM works in automatic measurement mode with low speed, the probing speed of the probe is about $2 \mathrm{~mm} / \mathrm{sec}$, and the influence of measurement speed can be neglected [15].

For realizing computer automatic evaluation of measurement uncertainty, considering the influence of the main uncertainty sources, the general uncertainty analysis model of CMM oriented dimension measurement can be expressed as the following formula based on the MCM idea.

$$
y=Y+\delta_{E}+\delta_{R P}+\delta_{R D}+\delta_{T}+\delta_{O B J}+\delta_{V}
$$

Where, $y$ is the synthetic measurement result, $\delta_{E}$ is the influence of CMM indication error on the measurement result, $\delta_{R P}$ is the influence of measurement repeatability on the measurement result, $\delta_{R D}$ is the influence of measurement reproducibility on the measurement result, $\delta_{T}$ is the influence of temperature change on the measurement result, $\delta_{O B J}$ is the influence of measured workpieces inconsistence on the measurement result, and $\delta_{V}$ is the influence of measurement speed on the measurement result.

Obviously, due to different measurement personnel, there is a great deal of subjectivity in the acquisition of $Y$ and $\delta_{R P}$, which will directly affect the evaluation result.

Therefore, the following alternative model is proposed.

$$
y=Y^{\prime}+\delta_{E}+\delta_{R D}+\delta_{T}+\delta_{O B J}
$$

In the formula, $Y^{\prime}$ is the average of the calculation results obtained by substituting a large number of computer simulation samples into the measurement model. The simulation samples are obtained by MCM computer simulation. They are based on one actual measurement sample, taking the maximum permissible probe error of $\mathrm{CMM}, M P E_{p}$, as the constraint condition, and combining it with the direction cosine of the actual measuring point. Thus, $Y^{\prime}$ contains the effect of measurement repeatability, and it can reflect the randomness of detection more realistically. Moreover, the actual measurement process is carried out in the automatic low-speed measurement mode of the CMM, and the influence of the measurement speed on the measurement results can be ignored. The large sample point sets obtained by MCM simulation are based on the actual measurement sample, so the influence of measurement speed on the measurement results can also be ignored.

\section{EVALUATION PROCESS OF MEASUREMENT UNCERTAINTY}

Taking CMM dimensional measurement as an example, the schematic diagram of the measurement evaluation process of the proposed SVCMM method is shown in Fig.1., and the specific evaluation flow chart is shown in Fig.2.

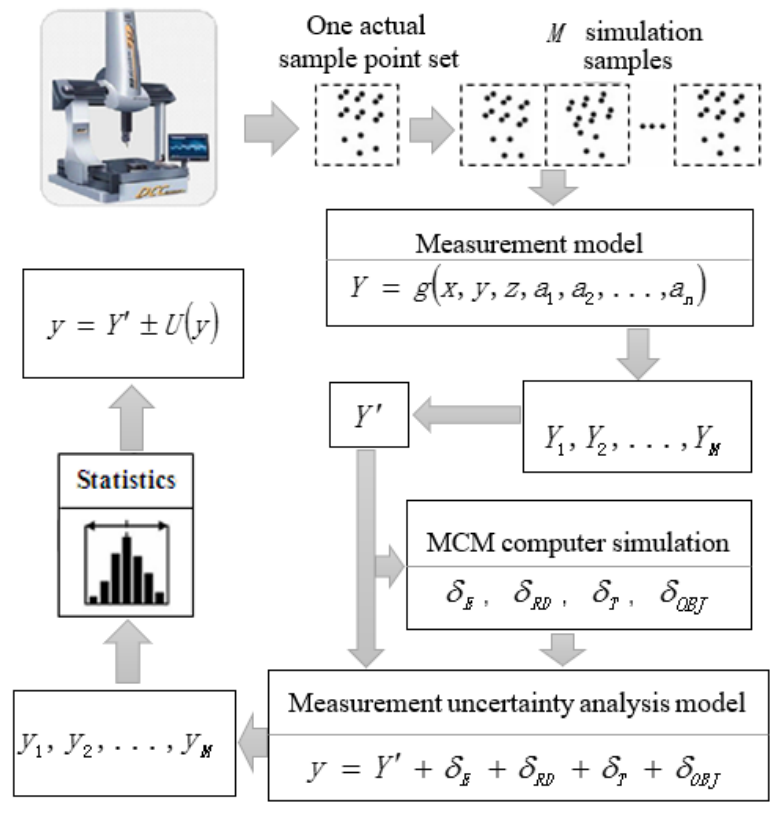

Fig.1. The schematic diagram of the SVCMM evaluation process for CMM dimensional measurement task.

As shown in Fig.2., the steps marked with "*" reflect the difference between the SVCMM evaluation and the traditional MCM evaluation.

In the traditional MCM evaluation, the single measurement result or the average value of multiple repeated measurements is used as the best estimate, and taken into the uncertainty model for synthesis. While in the SVCMM evaluation process, the actual sample points obtained in one measurement cycle are taken as the basis. According to the acceptance or reinspection report of CMM, the MCM computer simulation is used to fully reflect the 
probe randomness. A large number of simulation samples are obtained and taken into the measurement model. The average of the calculated results is taken as the best estimate and used to participate in the uncertainty synthesis.

It is visible that the SVCMM evaluation method can not only reflect the impact of detection randomness on the uncertainty evaluation results more fully, but also help the CMM users to obtain reasonable uncertainty evaluation results through one measurement cycle.

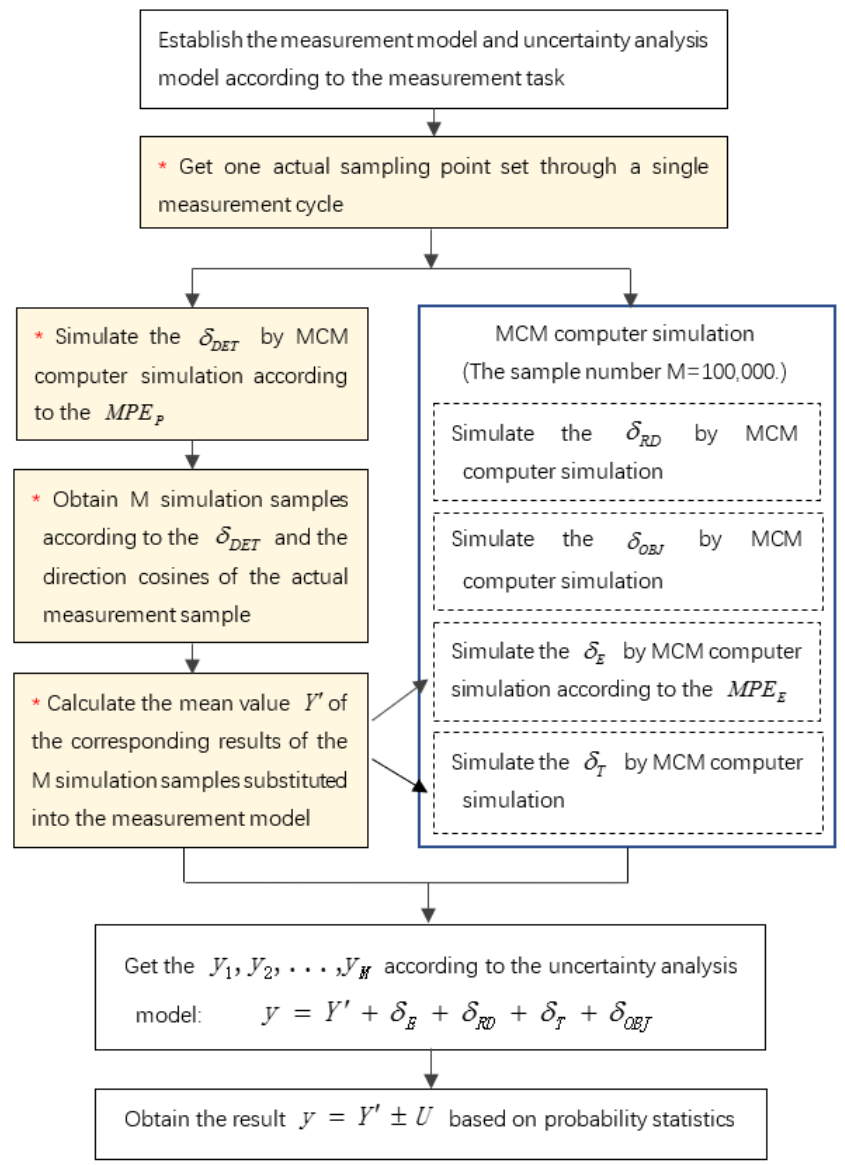

Fig.2. The SVCMM evaluation procedure of measurement uncertainty for CMM dimensional measurement task.

In order to guarantee the reliability of the uncertainty estimation, it is assumed that the effects of each error are uniformly distributed. The meaning and distribution characteristics of the variables in MCM simulation are shown in Table 1.

In Table 1., $M P E_{E}$ and $M P E_{P}$ are respectively the maximum permissible indication error and the maximum permissible probe error of CMM, which are provided in the acceptance or reinspection report of CMM. $\alpha_{W}$ and $\Delta \alpha_{W}$ are the thermal expansion coefficient of workpiece and the half width of its change, respectively. $\alpha_{M}$ and $\Delta \alpha_{M}$ are the thermal expansion coefficient of CMM grating ruler and the half width of its change, respectively. $\Delta T$ is the half width of the actual measurement temperature change from the standard $20^{\circ} \mathrm{C} . a_{R D}$ is the maximum effect of the measurement reproducibility according to the experience; $a_{O B J}$ is the maximum shape deviation obtained by experience or calibration measurement.

Table 1. The meaning and distribution characteristics of the variables in MCM simulation.

\begin{tabular}{|l|l|l|}
\hline symbol & \multicolumn{1}{|c|}{ Meaning } & \multicolumn{1}{c|}{$\begin{array}{c}\text { Distribution } \\
\text { characteristics }\end{array}$} \\
\hline$\delta_{D E T}$ & $\begin{array}{l}\text { the influence of probe } \\
\text { detection error }\end{array}$ & $U\left(-M P E_{P}, M P E_{P}\right)$ \\
\hline$\delta_{E}$ & $\begin{array}{l}\text { the influence of CMM } \\
\text { indication error }\end{array}$ & $U\left(-M P E_{E}, M P E_{E}\right)$ \\
\hline$\alpha_{W}^{\prime}$ & $\begin{array}{l}\text { the thermal expansion } \\
\text { coefficient of workpiece }\end{array}$ & $U\left(\alpha_{W}-\Delta \alpha_{W}, \alpha_{W}+\Delta \alpha_{W}\right)$ \\
\hline$\alpha_{M}^{\prime}$ & $\begin{array}{l}\text { the thermal expansion } \\
\text { coefficient of CMM } \\
\text { grating ruler }\end{array}$ & $U\left(\alpha_{M}-\Delta \alpha_{M}, \alpha_{M}+\Delta \alpha_{M}\right)$ \\
\hline$\Delta T^{\prime}$ & $\begin{array}{l}\text { the measurement } \\
\text { temperature change } \\
\text { from the standard } 20^{\circ} \mathrm{C}\end{array}$ & $U(-\Delta T, \Delta T)$ \\
\hline$\delta_{R D}$ & $\begin{array}{l}\text { the influence of } \\
\text { measurement } \\
\text { reproducibility }\end{array}$ & $U\left(-a_{R D}, a_{R D}\right)$ \\
\hline$\delta_{O B J}$ & $\begin{array}{l}\text { the influence of } \\
\text { measured workpieces } \\
\text { inconsistence }\end{array}$ & $U\left(-a_{O B J}, a_{O B J}\right)$ \\
\hline
\end{tabular}

As shown in Fig.2., $M$ samples of $\delta_{D E 71}(i=1,2, \ldots, M)$ can be obtained using the MCM method to simulate the influence of detection error in $M$ measurement cycles. According to the $\delta_{D E T \mathrm{~T}}$ and the direction cosines of the actual sample points obtained in one measurement cycle, the influence of detection error on the measurement points can be converted to the three axis directions $x, y, z$. Then, the new $M$ simulation samples can be obtained.

Assuming that one actual sample point is $P(x, y, z)$ and its direction cosines are $(i, j, k)$, the corresponding new point $P^{\prime}\left(x^{\prime}, y^{\prime}, z^{\prime}\right)$ can be obtained by (4), which has added the influence of CMM probe error according to $\delta_{D E T_{1}}$.

$$
\left[\begin{array}{c}
x^{\prime} \\
y^{\prime} \\
z^{\prime}
\end{array}\right]=\left[\begin{array}{c}
x \\
y \\
z
\end{array}\right]+\left[\begin{array}{ccc}
\delta_{D E T} & 0 & 0 \\
0 & \delta_{D E T} & 0 \\
0 & 0 & \delta_{D E T}
\end{array}\right]\left[\begin{array}{c}
i \\
j \\
k
\end{array}\right]
$$

When simulating $\delta_{T}$, the influence of temperature change in the measurement process, it is important to notice the type of dimensional measurement. It can be expressed as (5) or (6) according to the measurement task belonging to internal or external dimension measurement.

$$
\begin{aligned}
& \delta_{T}=\delta_{\text {Tin }}=Y^{\prime} \cdot\left(\alpha_{M}^{\prime}-\alpha_{W}^{\prime}\right) \cdot \Delta T^{\prime} \\
& \delta_{T}=\delta_{\text {Tout }}=Y^{\prime} \cdot\left(\alpha_{W}^{\prime}-\alpha_{M}^{\prime}\right) \cdot \Delta T^{\prime}
\end{aligned}
$$


Finally, the $y_{1}, y_{2}, \ldots, y_{M}$ can be obtained by synthesis according to the measurement uncertainty model, and the evaluation results of measurement uncertainty can be obtained through probability distribution and statistical analysis. The standard uncertainty $u(y)$ is the standard deviation of $M$ samples. Arranging the $y_{1}, y_{2}, \ldots, y_{M}$ in ascending order, if given $P$ as the contain probability, the confidence interval of the measurement results can be estimated for $\left[y_{(1-P) M / 2}, y_{(1+P) M / 2}\right]$ When the interval is symmetric, the expanded uncertainty and coverage factor can be determined as:

$$
\begin{gathered}
U(y)=\left[y_{(1+P) M / 2}-y_{(1-P) M / 2}\right] / 2, \\
k=U(y) / u(y)
\end{gathered}
$$

and the final measurement result can be expressed as

$$
y=Y^{\prime} \pm U(y)
$$

\section{EVALUATION EXAMPLE}

Taking the cylinder thickness measurement of automobile air conditioning compressor as an example, the proposed SVCMM method was applied to the uncertainty evaluation.

According to the drawings, the thickness value of the measured workpieces is $50 \pm 0.02 \mathrm{~mm}$. The surface roughness can be ignored. The $\alpha_{W}$ is $23.2 \times 10^{-6} /{ }^{\circ} \mathrm{C}$, and the $\Delta \alpha_{W}$ is $1.0 \times 10^{-6} /{ }^{\circ} \mathrm{C}$. Micro-Hite 3D DCC, the CMM of Hexagon, was taken as the measuring equipment. Its $M P E_{E}$ is expressed as $(3.0+4.0 L / 1000) \mu \mathrm{m}$, and the $M P E_{P}$ is $3.5 \mu \mathrm{m}$. The $\alpha_{M}$ is $10.5 \times 10^{-6} /{ }^{\circ} \mathrm{C}$, and the $\Delta \alpha_{M}$ is $1.0 \times 10^{-6} /{ }^{\circ} \mathrm{C}$.

The least square measurement model of workpiece thickness is established such as (9), and the measurement uncertainty analysis model is as (10).

$$
\begin{gathered}
Y=\left|z_{k}-a x_{k}-b y_{k}-c\right| / \sqrt{a^{2}+b^{2}+1} \\
y=Y+\delta_{E}+\delta_{T}+\delta_{R D}+\delta_{O B J}
\end{gathered}
$$

The measurement process conformed to the operation specification and using conditions. CMM automatic measurement was used under the condition of low speed. The workpiece was placed on the measuring platform in a natural state. The probe head was configured with 4BY30, and the direction angle was T1A0B0. The measuring head was calibrated, and the workpiece coordinate system was established according to the drawing reference.

For the convenience of measurement, in one complete measurement cycle, four roughly symmetrical points $P_{i}$ $(i=1,2,3,4)$ were taken on one measurement plane, one point $P_{k}$ was taken from another relative end face of the workpiece. In addition, the biggest flatness of the workpiece datum was tested as $0.001 \mathrm{~mm}$ using the CMM measurement software, and the maximum repeatability error was $0.0023 \mathrm{~mm}$ according to the measurement experience. The temperature change was about $20 \pm 1^{\circ} \mathrm{C}$ in the measurement process. The coordinate data and direction cosines of the points $P_{i}$ and $P_{k}$ are shown in Table 2. and Table 3 .

Table 2. The coordinate data and direction cosines of measured points $P_{i}$.

\begin{tabular}{|c|c|c|c|c|c|c|}
\hline point & $\boldsymbol{x}$ & $\boldsymbol{y}$ & $\boldsymbol{z}$ & $\boldsymbol{i}$ & $\boldsymbol{j}$ & $\boldsymbol{k}$ \\
\hline$P_{1}$ & -73.0747 & 128.3511 & -0.0018 & 0 & 0 & 1 \\
\hline$P_{2}$ & $\begin{array}{c}119.414 \\
5\end{array}$ & 86.9125 & -0.0028 & 0 & 0 & 1 \\
\hline$P_{3}$ & 76.5564 & -112.1250 & -0.0009 & 0 & 0 & 1 \\
\hline$P_{4}$ & -78.0221 & -78.8322 & -0.0017 & 0 & 0 & 1 \\
\hline
\end{tabular}

Table 3. The coordinate data and direction cosine of measured point $P_{k}$.

\begin{tabular}{|c|c|c|l|l|l|}
\hline Measured $\boldsymbol{x}$ & Measured $\boldsymbol{y}$ & Measured $\boldsymbol{z}$ & $\boldsymbol{i}$ & $\boldsymbol{j}$ & $\boldsymbol{k}$ \\
\hline 30.5497 & -46.4639 & 50.0106 & 0 & 0 & 1 \\
\hline
\end{tabular}

The traditional GUM method, MCM method and the proposed SVCMM method are used to evaluate the uncertainty of the measurement results, respectively. The sample number of computer simulations is taken as $M=100,000$, and the confidence probability is assumed as $P=95 \%$. The evaluation results are shown in Table 4 . The comparison of the distributions and $95 \%$ confidence interval limits of the results is shown in Fig.3. The comparison of the best estimate values and the confidence intervals of the results is shown in Fig.4.

Table 4. The results comparison of the workpiece thickness evaluated by GUM, MCM and SVCMM methods.

\begin{tabular}{|l|c|c|c|c|c|}
\hline Method & $\begin{array}{c}y \\
(\mathrm{~mm})\end{array}$ & $\begin{array}{c}u(y) \\
(\mathrm{mm})\end{array}$ & $k$ & $\begin{array}{c}U_{95} \\
(\mathrm{~mm})\end{array}$ & $\begin{array}{c}\text { The } \\
\text { confidence } \\
\text { interval } \\
\text { satisfied } \\
\text { for } \\
=95 \%\end{array}$ \\
\hline GUM & 50.0120 & 0.00240 & 2 & 0.0048 & $\begin{array}{c}{[50.0072,} \\
50.0168]\end{array}$ \\
\hline MCM & 50.0120 & 0.00237 & 1.89 & 0.0045 & $\begin{array}{c}{[50.0050} \\
50.0187]\end{array}$ \\
\hline SVCMM & 50.0122 & 0.00237 & 1.89 & 0.0045 & $\begin{array}{c}{[50.0055,} \\
50.0190]\end{array}$ \\
\hline
\end{tabular}

It can be seen by comparison that the evaluation results of the three methods are more consistent. Judging by the accepted GUM evaluation results, it can be concluded that the SVCMM evaluation method is feasible and the evaluation results are effective. 


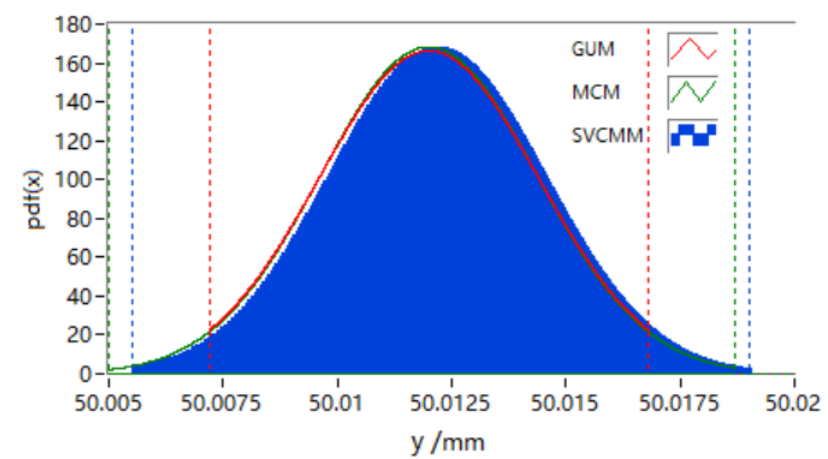

Fig.3. The distributions and $95 \%$ confidence interval limits evaluated by GUM (red, line), MCM (green, line) and SVCMM (blue, histogram) methods.

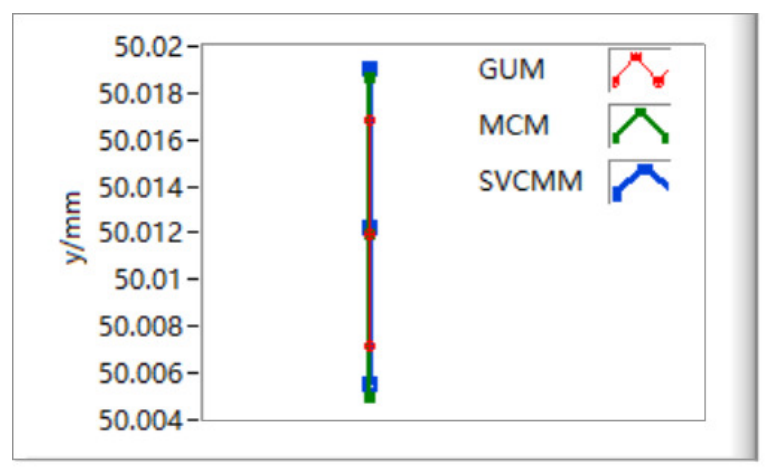

Fig.4. The best estimate values and the confidence intervals evaluated by GUM (red), MCM (green) and SVCMM (blue) methods.

\section{CONCLUSION}

Taking dimension measurement as an example, this paper presents a method of uncertainty modeling and evaluation of CMM task oriented measurement.

The established uncertainty analysis model is easy to understand and easy to expand. For different geometry measurements, the error items that affect the measurement results can be changed according to the actual situation. The proposed SVCMM evaluation method is applied on the basis of the actual measurement points, so it is suitable for all dimensional measurement tasks. Especially, the SVCMM method is based on MCM computer simulation to simulate the random sampling of errors, which is more in line with the actual impact of the error. Moreover, this method is easy to carry out by computer programming, which can improve the evaluation efficiency and realize the intelligent evaluation.

The evaluation example shows that the proposed uncertainty modeling and evaluation method of CMM task oriented measurement is effective and feasible, it can help the CMM users to complete the reasonable evaluation of the measurement uncertainty through a single measurement process, and the proposed method is economical and practical. Considering the dynamic impact on measurement, further research on the measurement uncertainty evaluation of CMM at different speeds will be carried out to improve the evaluation method.

\section{ACKNOWLEDGMENT}

This research is supported by Research Foundation of Shenzhen (No. JCYJ2016 0301153317415), Natural Science Foundation of Guangdong Province (No. 2014 A030310318), and National Key Research and Development Plan of China (No. 2016 YFF0203801).

\section{REFERENCES}

[1] International Organization for Standardization. (2003). Measurement management systems - Requirements for measurement processes and measuring equipment. ISO 10012:2003.

[2] International Organization for Standardization. (2011). Geometrical Product Specifications (GPS) -Inspection by measurement of workpieces and measuring equipment -- Part 2: Guide for the estimation of uncertainty in GPS measurement, in calibration of measuring equipment and in product verification. ISO 14253-2:2011.

[3] Zhang, G. (1999). Three-coordinate Measuring Machine. China: Tianjin University Press.

[4] Chen, X., Li, H., Yang, Q., Wang, H., Cheng, Y. (2015).Task-oriented measurement uncertainty evaluation of coordinate measuring machine. Acta Metrologica Sinica, 36 (6), 579-583.

[5] Joint Committee for Guides in Metrology. (2008). Evaluation of measurement data -- Guide to the expression of uncertainty in measurement. JCGM 100:2008 (GUM 1995 with minor corrections).

[6] Štubňa, I., Šín, P., Trník, A., Vozár, L. (2014). Measuring the flexural strength of ceramics at elevated temperatures - an uncertainty analysis. Measurement Science Review, 14 (1), 35-40.

[7] Azpurua, M., Tremola, C., Paez, E. (2011). Comparison of the GUM and Monte Carlo methods for the uncertainty estimation in electromagnetic compatibility testing. Progress in Electromagnetics Research B, 34, 125-144.

[8] Joint Committee for Guides in Metrology. (2008). Evaluation of measurement data--Supplement 1 to the "Guide to the expression of uncertainty in measurement" -- Propagation of distributions using a Monte Carlo method. JCGM 101:2008.

[9] State Administration of Quality Supervision, Inspection and Quarantine. (2012). Monte Carlo method for evaluation of measurement uncertainty. JJF 1059.2-2012.

[10] Wilhelm, R.G., Hocken, R., Schwenke, H. (2001). Task specific uncertainty in coordinate measurement. CIRP Annals, 50 (2), 553-563.

[11] Hu, Y., Yang, Q., Sun, X. (2012). Design, implementation, and testing of advanced virtual 
coordinate-measuring machines. IEEE Transactions on Instrumentation \& Measurement, 61 (5), 1368 1376.

[12] Sadek, J., Gaska, A. (2012). Evaluation of coordinate measurement uncertainty with use of virtual machine model based on Monte Carlo method. Measurement, 45 (6), 1564-1575.

[13] Jakubiec, W., Płowucha, W., Starczak, M. (2012). Analytical estimation of coordinate measurement uncertainty. Measurement, 45 (10), 2299-2308.

[14] Iakovidis, S., Apostolidis, C., Samaras, T. (2015). Application of the Monte Carlo method for the estimation of uncertainty in radiofrequency field spot measurements. Measurement Science Review, 15 (2), 72-76.

[15] Hexagon Measurement Technology Company. (2008). The Practical Coordinate Measuring Technology. China: Chemical Industry Press.

Received July 27, 2017. Accepted September 22, 2017. 